\section{Improved deterministic measurement model for consumer-grade accelerometers}

\section{B. Barshan ${ }^{\bowtie}$ and G. Seçer}

Deterministic error modelling, calibration and model parameter estimation of consumer-grade accelerometers is considered and improvement to the traditionally used measurement model is proposed. Calibration experiments on a flight motion simulator are performed for experimental verification. Model parameters are estimated using the Levenberg-Marquardt optimisation algorithm. Residual errors are considerably reduced as a result of the improved measurement model.

Introduction: With developments in microelectromechanical systems, the size, weight and cost of accelerometers have decreased considerably during the last two decades, opening up new application areas for their use [1].

An accelerometer detects specific force, which is proportionate to the acceleration relative to an inertial reference frame along its axis/axes of sensitivity. Accelerometer measurements often deviate from the ground truth since they suffer from various error types, which can be constant or time varying. As a result of double integrating their output to obtain linear position estimates, even very small errors accumulate very quickly and the output tends to drift. Proper characterisation, modelling and calibration of consumer-grade accelerometers is essential to obtain accurate position estimates and extend the time period over which these devices can be used stand-alone or in aided mode. Deterministic and stochastic modelling of inertial sensors are usually treated separately. Here, we focus on the deterministic calibration of accelerometers and propose an improvement to the traditional measurement model used in $1 \boldsymbol{g}$ tests. The effectiveness of the model is verified through calibration experiments and the results are compared with those of the traditionally used model.

The following notation is used throughout. Any vector $\boldsymbol{v}$ expressed with respect to a coordinate frame $f$ is denoted by $\boldsymbol{v}^{f}$, and the direction cosine matrix, denoted by $\boldsymbol{C}_{f_{1}}^{f_{2}}$, representing the rotational transformation between two coordinate frames $f_{1}$ and $f_{2}$, transforms a vector $v^{f_{1}}$ from frame $f_{1}$ to $f_{2}$ as $\boldsymbol{v}^{f_{2}}=\boldsymbol{C}_{f_{1}}^{f_{2}} \boldsymbol{v}^{f_{1}}$. Orthonormal basis vectors of the $x, y$ and $z$ axes of a given frame $f$ are, respectively, denoted by $i^{f}, \dot{j}^{f}$ and $\boldsymbol{k}^{f}$. Several coordinate frames need to be defined:

North-east-down (NED) frame is the Earth's frame of reference whose unit vectors lie along the north, east and down directions.

Platform base frame $(p)$ is an orthogonal frame fixed to the base of the rotating platform onto which the accelerometers are mounted and does not move together with the platform.

Sensor enclosure frame $(q)$ corresponds to the orthogonal axes of the sensor mechanical casing. Because of manufacturing tolerances and packaging issues, it cannot be perfectly aligned with the sensitivity axes of the sensor (the $s$ frame) in practice. This frame moves together with the platform onto which the accelerometers are attached.

Orthogonal sensor sensitivity frame $(r)$ is the idealised, orthogonal sensor sensitivity frame.

Non-orthogonal sensor sensitivity frame (s) represents the set of actual sensitivity axes of the accelerometer. Deviation from orthogonality stems from manufacturing tolerances.

For the platform (a flight motion simulator [FMS]) that we use for the calibration tests, the $\boldsymbol{k}$ unit vectors of the NED and $p$ frames are coincident and their respective $\boldsymbol{i}$ and $\boldsymbol{j}$ unit vectors lie on the horizontal plane, making an angle $\beta$ with each other (Fig. 1). Thus, the two frames are related by a rotational transformation $\boldsymbol{C}_{\mathrm{NED}}^{p}$ about the $\boldsymbol{k}$ axis by $\beta$ :

$$
\boldsymbol{C}_{\mathrm{NED}}^{p}=\left[\begin{array}{ccc}
\cos \beta & \sin \beta & 0 \\
-\sin \beta & \cos \beta & 0 \\
0 & 0 & 1
\end{array}\right]
$$

The $\boldsymbol{C}_{p}^{q}$ represents the rotational transformation from frame $p$ to $q$ and can be expressed mathematically by a sequence of basic rotations as $\boldsymbol{C}_{p}^{q}=\boldsymbol{R}_{x}(\phi) \boldsymbol{R}_{y}(\gamma) \boldsymbol{R}_{z}(\psi)$, where $\phi, \gamma$ and $\psi$ are the rotation angles about the $x, y$ and $z$ axes of the $p$ frame, respectively.

The $\boldsymbol{C}_{q}^{r}$ is the package misalignment matrix that describes the rotational transformation from frame $q$ to $r$ that have imperfect alignment and is given by $\boldsymbol{C}_{q}^{r}=\boldsymbol{R}_{x}\left(\varepsilon_{x}\right) \boldsymbol{R}_{y}\left(\varepsilon_{y}\right) \boldsymbol{R}_{z}\left(\varepsilon_{z}\right)$, where $\varepsilon_{x}, \varepsilon_{y}$ and $\varepsilon_{z}$ are the mounting misalignment angles about the $x, y$ and $z$ axes of the $q$ frame, respectively.

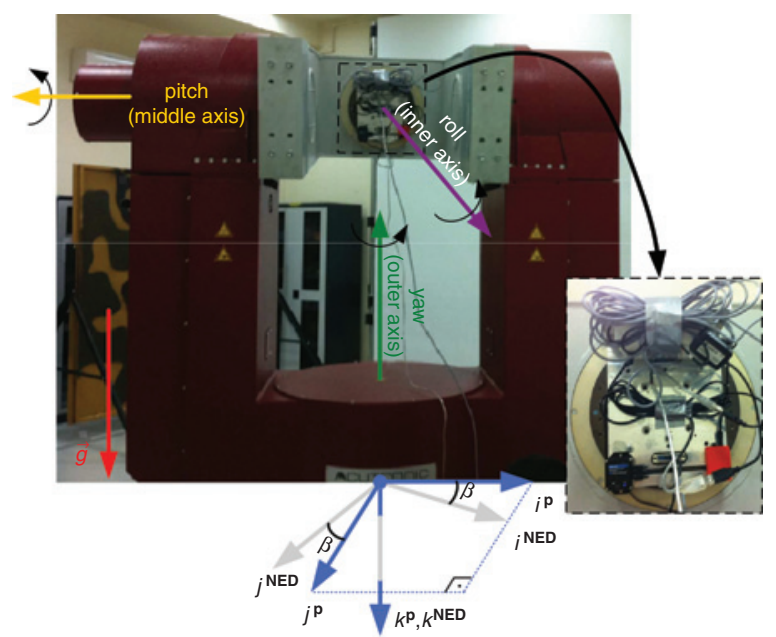

Fig. 1 ACUTRONIC FMS during calibration procedure Inset: Fixture plate onto which two accelerometers are attached

The non-orthogonalisation matrix $\boldsymbol{T}_{r}^{s}$ describes the kinematic transformation from frame $r$ to $s$ :

$$
\boldsymbol{T}_{r}^{s}=\left[\begin{array}{ccc}
1 & 0 & 0 \\
\sin \left(\alpha_{1}\right) & \cos \left(\alpha_{1}\right) & 0 \\
\cos \left(\alpha_{3}\right) & \sin \left(\alpha_{2}\right) \sin \left(\alpha_{3}\right) & \cos \left(\alpha_{2}\right) \sin \left(\alpha_{3}\right)
\end{array}\right]
$$

where $\alpha_{i}, i \in\{1,2,3\}$ are the sensor-to-sensor axis misalignment angles.

We also introduce a scale factor error matrix $\boldsymbol{S}$ that scales the output of each accelerometer axis by a different amount in general:

$$
\boldsymbol{S}=\left[\begin{array}{ccc}
S_{x} & 0 & 0 \\
0 & S_{y} & 0 \\
0 & 0 & S_{z}
\end{array}\right]
$$

When the input to the accelerometer is zero, the deviation of the output from the zero level is the bias error $\boldsymbol{b}=\left[b_{x} b_{y} b_{z}\right]^{\mathrm{T}}$ which may drift in time and change with the operating temperature of the sensor.

Traditional accelerometer measurement model: The traditionally used first-order measurement model of accelerometers is given by [2]

$$
\boldsymbol{a}_{\mathrm{m}}=(\boldsymbol{I}+\boldsymbol{S}) \boldsymbol{T}_{r}^{s} \boldsymbol{C}_{q}^{r} \boldsymbol{C}_{p}^{q} \boldsymbol{a}^{p}+\boldsymbol{b}+\boldsymbol{n}
$$

The $\boldsymbol{a}_{\mathrm{m}}$ is the acceleration measured along the sensitivity axes of the accelerometer (the $s$ frame), whereas the reference for the true value of the excitation signal $\boldsymbol{a}^{p}$ is the $p$ frame. The composite matrix multiplying $\boldsymbol{a}^{p}$ above represents a transformation from the $p$ to the $s$ frame and corrects for the scale factor error. Here, $\boldsymbol{I}$ is the $3 \times 3$ identity matrix and $\boldsymbol{n}$ is the additive stochastic measurement noise vector.

Improved accelerometer measurement model: We propose the following improvement to the measurement model:

$$
\boldsymbol{a}_{\mathrm{m}}=(\boldsymbol{I}+\boldsymbol{S}) \boldsymbol{T}_{r}^{s} \boldsymbol{C}_{q}^{r} \boldsymbol{C}_{p}^{q} \boldsymbol{C}_{\mathrm{NED}}^{p} \boldsymbol{a}^{\mathrm{NED}}+\boldsymbol{b}+\boldsymbol{n}
$$

The reference for the true acceleration $\boldsymbol{a}^{\mathrm{NED}}$ is the NED frame. The composite matrix multiplying $\boldsymbol{a}^{\mathrm{NED}}$ above represents a transformation from the NED to the $s$ frame and corrects for the scale factor error.

Note that in the traditional model, $\boldsymbol{C}_{\mathrm{NED}}^{p}$ is not included and when the FMS is at stationary angular positions, the true acceleration is taken as $\boldsymbol{g}^{p}$ instead of $\boldsymbol{g}^{\mathrm{NED}}$. The following approximation is made when using $\boldsymbol{g}^{p}$ : given that the $\boldsymbol{i}-\boldsymbol{j}$ planes of both $p$ and NED frames lie on the horizontal plane (perpendicular to $\boldsymbol{g}$ ) with coincident $\boldsymbol{k}$ unit vectors, as shown in Fig. 1 and described by (1), the main component of both $\boldsymbol{g}^{p}$ and $\boldsymbol{g}^{\text {NED }}$ lies along the $\boldsymbol{k}$ direction and the much smaller components along $\boldsymbol{i}^{p}, \boldsymbol{j}^{p}, \boldsymbol{i}^{\mathrm{NED}}$ and $\boldsymbol{j}^{\mathrm{NED}}$ can be neglected. Thus, in the traditional model, the excitation signal $\boldsymbol{g}^{p}$ is usually approximated by the third component of $\boldsymbol{g}^{\mathrm{NED}}$ and used in multi-position tests, resulting in some error.

Calibration experiments: We use ACUTRONIC's high-precision 3-DOF FMS (Fig. 1) to conduct multi-position tests for the deterministic calibration of the tri-axial accelerometers of two widely used consumergrade inertial measurement units: the 3DM-GX2 of MicroStrain [3] and 
the MTx of Xsens [4]. Both units are attached side by side to the fixture plate of the FMS, located on the shaft of the inner axis (Fig. 1 inset). A trajectory for the axes of the FMS is determined and programmed into the FMS controller computer. During the calibration procedure, both accelerometer outputs are sampled uniformly at $100 \mathrm{~Hz}$. We only consider the measurements acquired while the FMS is stationary at different angular positions to avoid additional disturbance on the measurements that might occur while the FMS is in motion.

Accelerometer measurement model parameter estimation: We use the model-based nonlinear Levenberg-Marquardt optimisation algorithm (LMA) [5] to estimate the measurement model parameters.

- In accordance with the error components described previously, the unknown parameter vector $\boldsymbol{\theta}$ for the improved model is given by:

$$
\boldsymbol{\theta}=\left[\begin{array}{lllllllllllll}
S_{x} & S_{y} & S_{z} & \alpha_{1} & \alpha_{2} & \alpha_{3} & \varepsilon_{x} & \varepsilon_{y} & \varepsilon_{z} & \beta & b_{x} & b_{y} & b_{z}
\end{array}\right]^{\mathrm{T}}
$$

- We form a single column vector of $3 N$ elements based on the accelerometer measurements:

$$
\boldsymbol{y}=\left[\begin{array}{llll}
\boldsymbol{a}_{\mathrm{m}}^{\mathrm{T}}[1] & \boldsymbol{a}_{\mathrm{m}}^{\mathrm{T}}[2] & \cdots & \boldsymbol{a}_{\mathrm{m}}^{\mathrm{T}}[N]
\end{array}\right]^{\mathrm{T}}
$$

Here, $\boldsymbol{a}_{\mathrm{m}}[k], k=1, \ldots, N$, denote the measurement vector of accelerometers at time sample $k$.

- Parametric form of the accelerometer output, acquired from the first two terms on the right-hand side of (5), is also represented as a vector with $3 N$ elements:

$$
\boldsymbol{F}(\boldsymbol{\theta})=\left[\begin{array}{lll}
\boldsymbol{F}_{1}^{\mathrm{T}}\left[\boldsymbol{g}^{\mathrm{NED}}, \boldsymbol{\theta}\right] & \cdots & \boldsymbol{F}_{N}^{\mathrm{T}}\left[\boldsymbol{g}^{\mathrm{NED}}, \boldsymbol{\theta}\right]
\end{array}\right]^{\mathrm{T}}
$$

where $\boldsymbol{F}_{k}^{\mathrm{T}}\left[\boldsymbol{g}^{\mathrm{NED}}, \boldsymbol{\theta}\right]$ are obtained by using $\boldsymbol{C}_{p}^{q}[k], k=1, \ldots, N$, which represent the FMS orientations when the FMS is stationary.

- The fitness function to be minimised by the LMA is $\|\boldsymbol{y}-\boldsymbol{F}(\boldsymbol{\theta})\|$.

For the ideal accelerometer that does not require any calibration (without any misalignment, orthogonalisation, scale factor or bias errors), the ideal calibration parameter vector $\boldsymbol{\theta}_{\mathrm{o}}$ is given by:

$$
\boldsymbol{\theta}_{\circ}=\left[\begin{array}{lllllllllllll}
0 & 0 & 0 & 0 & 0 & \frac{\pi}{2} & 0 & 0 & 0 & 0 & 0 & 0 & 0
\end{array}\right]^{\mathrm{T}}
$$

with all its parameters being equal to zero, except for $\alpha_{3}=\pi / 2$.

Considering the centrifugal acceleration of the Earth, $\boldsymbol{g}^{\mathrm{NED}}$ at the location where the experiments are conducted can be calculated from [6]:

$$
\boldsymbol{g}^{\mathrm{NED}}=\boldsymbol{g}-\frac{(R+\ell)\left\|\boldsymbol{\omega}^{\mathrm{NED}}\right\|^{2}}{2}\left[\begin{array}{lll}
\sin 2 \Lambda & 0 & (1+\cos 2 \Lambda)
\end{array}\right]^{\mathrm{T}}
$$

where $\boldsymbol{g}=\left[\begin{array}{lll}0 & 0 & 9.80665\end{array}\right]^{\mathrm{T}}$ is the standard gravity vector and $\boldsymbol{\omega}^{\mathrm{NED}}, R, \ell$ and $\Lambda$ denote the Earth's turn rate vector with respect to the NED frame, the radius of the Earth, altitude with respect to sea level and the latitude angle that changes between $-90^{\circ}$ and $90^{\circ}$, respectively. The $\boldsymbol{g}^{\mathrm{NED}}$ vector is calculated as $\boldsymbol{g}^{\mathrm{NED}}=\left[\begin{array}{lll}-0.0167 & 0 & 9.7782\end{array}\right]^{\mathrm{T}} \mathrm{m} / \mathrm{s}^{2}$.

For the traditional model, the procedure is very similar. The only difference is that $\boldsymbol{\theta}$ and $\boldsymbol{\theta}_{\circ}$ are reduced by one dimension $(\beta)$ and (4) is used instead of (5) so that $\boldsymbol{g}^{\text {NED }}$ in (8) is replaced by $\boldsymbol{g}^{p}$.

Table 1: Estimation errors of accelerometers without calibration and with calibration using traditional and improved measurement models

\begin{tabular}{|c|c|c|c|c|}
\hline \multicolumn{2}{|c|}{ Units: $\mathrm{m} / \mathrm{s}^{2}$} & $\begin{array}{c}\left\|\boldsymbol{y}-\boldsymbol{F}\left(\boldsymbol{\theta}_{\circ}\right)\right\| \\
\text { without }\end{array}$ & $\begin{array}{c}\left\|\boldsymbol{y}-\boldsymbol{F}\left(\boldsymbol{\theta}^{*}\right)\right\| \\
\text { traditional }\end{array}$ & $\begin{array}{c}\left\|\boldsymbol{y}-\boldsymbol{F}\left(\boldsymbol{\theta}^{*}\right)\right\| \\
\text { improved }\end{array}$ \\
\hline \multirow{3}{*}{ MicroStrain } & $x$-axis & 19.02 & 3.40 & 2.87 \\
\cline { 2 - 5 } & $y$-axis & 20.24 & 3.04 & 2.67 \\
\cline { 2 - 5 } & $z$-axis & 19.92 & 2.83 & 2.12 \\
\hline \multirow{3}{*}{ Xsens } & $x$-axis & 5.71 & 2.46 & 2.21 \\
\cline { 2 - 5 } & $y$-axis & 9.48 & 2.76 & 2.52 \\
\cline { 2 - 5 } & $z$-axis & 6.94 & 2.31 & 1.76 \\
\hline
\end{tabular}

The error values without any calibration, and those with calibration using the traditional and improved models, are provided in Table 1. These correspond to the square root of the sum of the squared errors for each accelerometer axis. The $\boldsymbol{F}\left(\boldsymbol{\theta}_{\mathrm{o}}\right)$ in the second column of Table 1 is calculated by using the $\boldsymbol{\theta}_{\circ}$ in (9) (i.e. assuming an ideal accelerometer). Since $\boldsymbol{F}_{k}\left[\boldsymbol{g}^{\mathrm{NED}}, \boldsymbol{\theta}_{\circ}\right]=\boldsymbol{g}^{\mathrm{NED}}$ for $k=1, \ldots, N$, the elements of $\boldsymbol{F}\left(\boldsymbol{\theta}_{\circ}\right)$ in (8) are simplified to $\left(\boldsymbol{g}^{\mathrm{NED}}\right)^{\mathrm{T}}$ in this case. The $\boldsymbol{\theta}^{*}$ in the third and fourth columns of the Table is the parameter vector optimised using the LMA. With the improved measurement model proposed here, the errors are reduced by $18 \%$ for the MicroStrain and $14 \%$ for the Xsens accelerometers at the expense of estimating an additional parameter $\beta$.

The estimated model parameters of the two accelerometers are provided in Table 2 . The true value of $\beta$, measured by using a gyrotheodolite, was $89^{\circ}=1.553 \mathrm{rad}$ during the experiments. The estimated $\beta$ values given in the Table for the two accelerometers are close to the true value. The difference between the two estimates is about $7^{\circ}$, indicating their consistency. We note that rotation by the angle $\beta$ about the $z$-axis of the NED frame does not affect the vertical component of the gravity while the 600-times-smaller horizontal component becomes affected. The value of the horizontal gravity component is $-0.0167 \mathrm{~m} / \mathrm{s}^{2}$; thus comparable with the noise levels of the two accelerometers with standard deviations of $0.013 \mathrm{~m} / \mathrm{s}^{2}$ (for MicroStrain) and $0.012 \mathrm{~m} / \mathrm{s}^{2}$ (for Xsens). The low signal-to-noise ratio makes estimation of $\beta$ difficult. We have also run the LMA using the true value of $\beta$ and have found the error reduction to be

\begin{tabular}{|c|c|}
\hline MicroStrain - traditional & MicroStrain -improved \\
\hline $\operatorname{diag}(\boldsymbol{S})=\left[\begin{array}{lll}0.0008 & -0.0001 & 0.0008\end{array}\right]$ & $\operatorname{diag}(\boldsymbol{S})=\left[\begin{array}{lll}0.002 & 0.001 & 0.002\end{array}\right]$ \\
\hline$\alpha_{1}=0.029^{\circ} \alpha_{2}=0.123^{\circ} \alpha_{3}=90.026^{\circ}$ & $\alpha_{1}=0.030^{\circ} \alpha_{2}=0.077^{\circ} \alpha_{3}=89.98^{\circ}$ \\
\hline$\varepsilon_{x}=1.081^{\circ} \varepsilon_{y}=-0.133^{\circ} \varepsilon_{z}=0.323^{\circ}$ & $\varepsilon_{x}=1.031^{\circ} \varepsilon_{y}=-0.181^{\circ} \varepsilon_{z}=0.308^{\circ}$ \\
\hline \multirow[t]{2}{*}{$\boldsymbol{b}=[-0.094-0.0130 .049]^{\mathrm{T}}\left(\mathrm{m} / \mathrm{s}^{2}\right)$} & 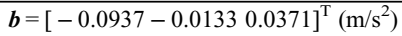 \\
\hline & $\beta=1.7231(\mathrm{rad})$ \\
\hline Xsens - traditional & Xsens-improved \\
\hline $\operatorname{diag}(\boldsymbol{S})=\left[\begin{array}{lll}0.0008 & 0.0008 & 0.0008\end{array}\right]$ & $\operatorname{diag}(\boldsymbol{S})=\left[\begin{array}{lll}0.002 & 0.002 & 0.002\end{array}\right]$ \\
\hline$\alpha_{1}=-0.043^{\circ} \alpha_{2}=0.093^{\circ} \alpha_{3}=89.993^{\circ}$ & $\alpha_{1}=-0.043^{\circ} \alpha_{2}=0.049^{\circ} \alpha_{3}=89.95^{\circ}$ \\
\hline$\varepsilon_{x}=0.485^{\circ} \varepsilon_{y}=0.002^{\circ} \varepsilon_{z}=-0.292^{\circ}$ & $\varepsilon_{x}=0.438^{\circ} \varepsilon_{y}=-0.049^{\circ} \varepsilon_{z}=-0.295^{\circ}$ \\
\hline \multirow[t]{2}{*}{$\boldsymbol{b}=[-0.003-0.001-0.002]^{\mathrm{T}}\left(\mathrm{m} / \mathrm{s}^{2}\right)$} & $\boldsymbol{b}=[-0.0028-0.00080 .0101]^{\mathrm{T}}\left(\mathrm{m} / \mathrm{s}^{2}\right)$ \\
\hline & $\beta=1.6053(\mathrm{rad})$ \\
\hline
\end{tabular}
about the same and the remaining parameter estimates to be very close to those reported in Table 2 .

Table 2: Estimated calibration parameters of accelerometers according to traditional and improved models

Conclusion: We have addressed the deterministic error modelling, calibration and parameter estimation of consumer-grade accelerometers. We have proposed an improvement to the traditional deterministic model of accelerometers and estimated the measurement model parameters using the LMA, based on experimentally acquired data through multi-position tests. Residual errors were considerably reduced compared with those obtained with the traditional model. Substantial improvement in the model fit makes the stand-alone and aided use of consumer-grade accelerometers possible for longer durations.

(C) The Institution of Engineering and Technology 2016

Submitted: 21 August 2015 E-first: 1 March 2016

doi: $10.1049 /$ el.2015.2967

One or more of the Figures in this Letter are available in colour online.

B. Barshan and G. Seçer (Department of Electrical and Electronics Engineering, Bilkent University, Ankara 06800, Turkey)

凶-mail: billur@ee.bilkent.edu.tr

\section{References}

1 Avvenuti, M., Casella, A., and Cesarini, D.: 'Using gait symmetry to virtually align a triaxial accelerometer during running and walking', Electron. Lett., 2013, 49, pp. 120-121

$2 \mathrm{Li}, \mathrm{X}$., and Li, Z.: 'A new calibration method for tri-axial field sensors in strap-down navigation systems', Meas. Sci. Technol., 2012, 23, p. 105105

3 MicroStrain, Williston, VT, USA: 3DM-GX2 ${ }^{\mathbb{R}}$ Microstrain, 2015. Available at http://www.microstrain.com/inertial/3DM-GX2

4 Xsens Tech. BV: Enschede, The Netherlands, MTi and MTx User Manual and Technical Documentation, 2015. Available at http://www.xsens.com

5 Press, W.H., Flannery, B.P., Teukolsky, S.A., and Vetterling, W.T.: 'Numerical recipes in C: the art of scientific computing' (Cambridge University Press, Cambridge, UK, 1992, 2nd edn), pp. 681-688

6 Titterton, D.H., and Weston, J.L.: 'Strapdown inertial navigation technology' (The American Institute of Aeronautics and Astronautics, Reston, VA, USA, 1979, 2nd edn) 